\title{
THE CALAR ALTO DEEP IMAGING SURVEY: FIRST RESULTS
}

\author{
E. THOMMES, K. MEISENHEIMER, \\ R. FOCKENBROCK, H. HIPPELEIN AND H.-J. RÖSER \\ Max-Planck-Institut für Astronomie, Heidelberg, Germany
}

\section{Introduction}

The Calar Alto Deep Imaging Survey (CADIS) is a very deep emission line survey using a Fabry-Pérot (FP), combined with deep broad- and mediumband photometry (for an overview see Hippelein et al. 1996). This survey is specifically designed to detect primeval galaxies, but it will in addition produce a large data base for investigations of faint galaxies at intermediate redshifts $(0.2<z<1.2)$. We present some first results from the initial data recorded with the CADIS strategy.

\section{Data}

These data were taken with the $2.2 \mathrm{~m}$ telescope at Calar Alto in the CADIS field $9 \mathrm{H}$. Due to delays in getting the $2 \mathrm{k} \times 2 \mathrm{k}$ CCDs, we employed a $1 \mathrm{k} \times 1 \mathrm{k}$ CCD (field of view $8 \times 8^{\prime}$ ). We got four FP settings in the wavelength region $814 \mathrm{~nm}$ to $818.5 \mathrm{~nm}$ (resolution=1.8nm). Every setting consists of 7 individual exposures of $1500 \mathrm{~s}$ integration. We reached a $5 \sigma$ detection limit of $S_{\text {lim }}(5 \sigma) \approx 5 \times 10^{-20} \mathrm{~W} / \mathrm{m}^{2}$. To get an estimate of the continuum near the emission lines, we did exposures with a filter $\lambda / \Delta \lambda=812 / 17 \mathrm{~nm}\left(F_{\text {lim }}(5 \sigma) \approx 5.8 \times 10^{-21} \mathrm{~W} /\left(\mathrm{m}^{2} \mathrm{~nm}\right)\right)$. The FP exposures were supplemented by broad band exposures with the filters $B V$ (centered at $500 \mathrm{~nm}, 5 \sigma$ limit $\left.\approx 25^{m} 8\right), R_{c}\left(5 \sigma\right.$ limit $\left.\approx 25^{m} 0\right)$ and $I(5 \sigma$ limit $\left.\approx 23{ }^{m} 1\right)$. Further narrow band exposures with the filters $466 / 9\left(F_{\text {lim }}(5 \sigma) \approx\right.$ $\left.11 \times 10^{-21} \mathrm{~W} /\left(\mathrm{m}^{2} \mathrm{~nm}\right)\right), 612 / 10\left(F_{\text {lim }}(5 \sigma) \approx 11 \times 10^{-21} \mathrm{~W} /\left(\mathrm{m}^{2} \mathrm{~nm}\right)\right)$ and $614 / 28\left(F_{\text {lim }}(5 \sigma) \approx 7 \times 10^{-21} \mathrm{~W} /\left(\mathrm{m}^{2} \mathrm{~nm}\right)\right)$ enable to detect further emission lines of foreground objects. Emission line objects are selected by the requirement, that they have a $5 \sigma$ detection in at least one FP wavelength setting and that the line flux exceeds the continuum by more than $3.5 \sigma$. 


\section{Results}

In this exploratory CADIS data set we already found 147 emission line galaxies which satisfy the conditions mentioned above. 104 show at least a marginal detection in the BV band and are therefore classified as faint blue galaxies in the foreground. 74 of these emission line objects with a blue detection also show signals in the additional narrow band exposures. These allowed us to identify them as being either at $z \approx 0.24$ or $z \approx 0.63 / 0.68$. Of the 43 emission line objects without detection in the blue band, 35 show at least a marginal detection in one of the additional narrow band filters and therefore also could be identified as galaxies at these redshifts. From the remaining 8 galaxies 7 are promising candidates for Ly- $\alpha$ emitting PGs at $z \approx 5.7$. Three of these 7 candidates have no continuum flux shortward of the emission line at $\lambda \approx 816 \mathrm{~nm}$ and 4 candidates showed a marginal detection in our red filter $(R \leq 26.0)$. Five of the candidates are clearly resolved ( $\geq 2^{\prime \prime}$ FWHM), while two objects may be unresolved.
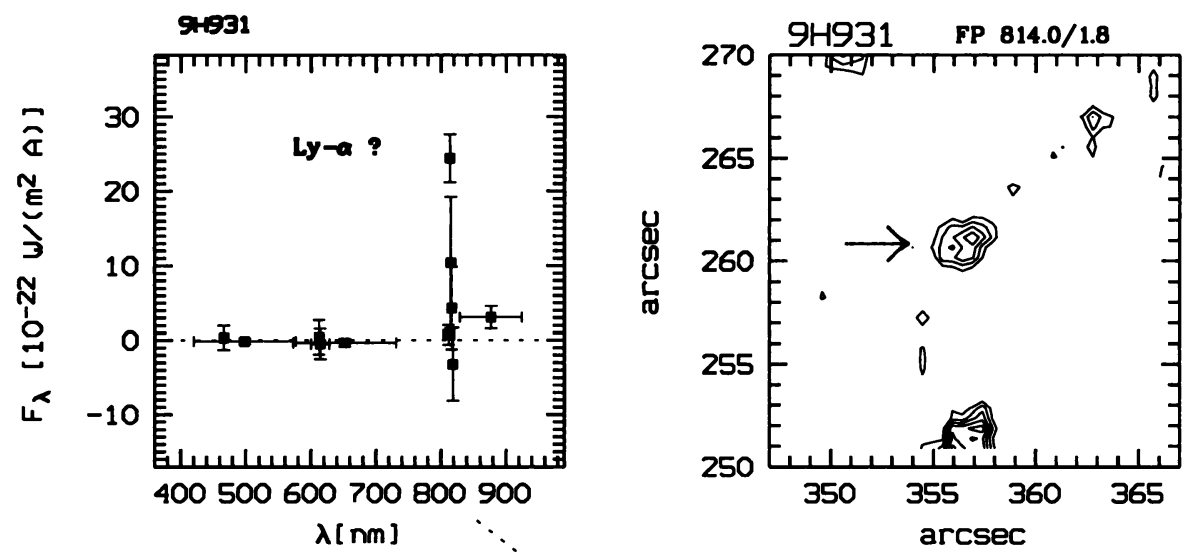

Figure 1. Photometric spectrum and conture plot of one of the 7 promising candidates for $L y$ - $\alpha$ emitting $P G s$ at $z=5.75$.

About $30 \%$ of the foreground emission line objects have no detectable continuum in the BV and R band. These objects are a new class of objects which are overlooked by broad band selected redshift surveys. We derived lower limits to the luminosity function from our data for the pure emission line objects in the redshift bins 0.24 and 0.64 . Although the covered spatial angle of the data presented here $\left(\approx 60 \square^{\prime}\right)$ is yet too small to draw definite conclusions these are in good agreement with the results of the AUTOFIB redshift survey (Colles et al. 1996) We expect that our sample of 7 candidates for primeval Ly- $\alpha$ emitting galaxies at $z \approx 5.7$ is still contaminated by several types of foreground objects. Statistical considerations indicate, 
that artifacts and emission line galaxies at $z=0.24$ and $z=0.63$ could hardly account for more than two of the candidates. The unknown fraction of galaxies at $z=1.2$ with strong [OII] line but undetectable blue continuum could however well make up for half of the candidates. Therefore, high $\mathrm{S} / \mathrm{N}$ ratio slit-spectroscopy at medium resolution $(\Delta \lambda \approx 0.5 \mathrm{~nm})$ and/or deeper continuum I, R and B band images are required to identify the true Ly- $\alpha$ galaxies among our candidates. We are pretty optimistic that at least some of the objects will, in fact, turn out to be at $z \approx 5.7$.

\section{References}

Colles et al., 1996, to appear in The Early Universe with the VLT, proceedings of ESOworkshop held in Garching bei München, Springer, Berlin, Heidelberg, New York, pp. 87.

Hippelein et al. 1996, this volume, 293 